\section{Kidney \\ Blood Pressure Research}

Kidney Blood Press Res 2015;40:355-365

DOI: $10.1159 / 000368510$

(C) 2015 S. Karger AG, Basel

www.karger.com/kbr

Accepted: April 30, 2015

$1423-0143 / 15 / 0404-0355 \$ 39.50 / 0$

This is an Open Access article licensed under the terms of the Creative Commons AttributionNonCommercial 3.0 Unported license (CC BY-NC) (www.karger.com/OA-license), applicable to the online version of the article only. Distribution permitted for non-commercial purposes only.

\title{
Tumor Necrosis Factor-Alpha and 8-Hydroxy-2'-Deoxyguanosine are Associated with Elevated Urinary Angiopoietin-2 Level in Type 2 Diabetic Patients with Albuminuria
}

\author{
Fang-Fang He $\mathrm{e}^{\mathrm{a}}$ Hui-Qing Li ${ }^{\mathrm{b}}$ Qing-Xian Huang ${ }^{\mathrm{c}}$ Qiu-Yuan Wang ${ }^{\mathrm{a}}$ Hua-Jun \\ Jiang $^{\text {a Shan Chen }}{ }^{\mathrm{a}}$ Hua Su ${ }^{\mathrm{a}}$ Chun Zhang ${ }^{\mathrm{a}}$ Yu-Mei Wang ${ }^{\mathrm{a}}$ \\ aDepartment of Nephrology; ${ }^{b}$ Department of Endocrinology, Union Hospital, Tongji Medical College, \\ Huazhong University of Science and Technology, Wuhan, Hubei; 'Department of Gastrointestinal \\ Surgery, Yantai Yuhuangding Hospital, Yantai, Shandong, China
}

\section{Key Words}

Angiopoietin $2 \cdot$ Tumor necrosis factor-alpha $\bullet$ 8-Hydroxy-2'-deoxyguanosine $\bullet$ Interleukin-18 - Superoxide dismutase

\begin{abstract}
Background/Aims: We previously showed that urine and serum Angiopoietin-2 (Ang-2) levels increased significantly with the degree of albuminuria in diabetes patients, but the reasons remain unclear. Consequently we aimed to determine whether there was an association between Ang-2, inflammatory cytokines (TNF- $\alpha$ and IL-18) and reactive oxygen species (8OHdG and SOD) in type 2 diabetes patients with albuminuria. Methods: This retrospective study evaluated 113 patients with type 2 diabetes and normoalbuminuria, microalbuminuria, or macroalbuminuria and 30 healthy controls. Serum and urine TNF- $\alpha$, IL-18 and 8-OHdG levels were measured by ELISA. Superoxide dismutase (SOD) activity was determined by spectrophotometry. Results: Serum and urine TNF- $\alpha$, IL-18 and 8-OHdG levels increased significantly with the degree of albuminuria, and were positively correlated with increased Ang-2. In contrast, SOD activity decreased with the degree of albuminuria and was negatively correlated with Ang-2. Multivariable linear regression analysis revealed that serum Ang-2 level was independently associated with serum levels of TNF- $\alpha(P<0.001), 8-\mathrm{OHdG}(P=0.001)$, and IL-18 $(P=0.003)$. Urinary Ang-2 level was independently associated with urinary TNF- $\alpha$
\end{abstract}

F.-F. He, H.-Q. Li and Q.-X. Huang contributed equally to this work and thus share first authorship. 


\section{Kidney Blood Pressure Research}

Kidney Blood Press Res 2015;40:355-365

\begin{tabular}{l|l}
\hline DOI: $10.1159 / 000368510$ & (c) 2015 S. Karger AG, Basel
\end{tabular}

Published online: June 20, 2015

www.karger.com $/ \mathrm{kbr}$

$(P<0.001)$ and 8-OHdG $(P=0.004)$ levels. Conclusion: TNF- $\alpha$ and 8-OHdG are associated with elevated urinary Angiopoietin-2 levels in type 2 diabetic patients with albuminuria.

Copyright (C 2015 S. Karger AG, Basel

\section{Introduction}

Angiopoietins comprise a family of vascular growth factors with angiopoietin-1 (Ang-1) and Ang-2 being the most studied [1]. They are thought to be involved in the pathogenesis of diabetic nephropathy (DN). Increased Ang-2 is associated with signs of renal damage such as albuminuria and decreased glomerular filtration rate [2-4]. Our previous study found that the levels of Ang- 2 were both elevated in the serum and urine of diabetic patients even in the absence of microalbuminuria, increased urinary Ang-2 was positively associated with the degree of albuminuria [5]. However, the mechanism for the elevation of Ang-2 is not clear.

Inflammatory cytokines are believed to participate in the development of DN, and there is evidence that inflammation and angiogenesis are mutually dependent [6-9]. Tumor necrosis factor (TNF)- $\alpha$ and interleukin (IL)-18 are considered to be the main regulators of inflammatory cytokines in DN [10-12]. Fiedler and Augustin have proposed that Ang2 triggers an inflammatory response in endothelial cells by promoting the activities of inflammatory cytokines [6]. Activation of an inflammatory response can trigger molecular cascades that result in leukocyte recruitment and transmigration. Ang-2, TNF- $\alpha$ and IL-18 are all involved in this process [6]. However, the relationships of Ang- 2 with levels of TNF- $\alpha$ and IL-18 in the serum and urine of DN patients are not clear.

Reactive oxygen species (ROS) are regarded as the common denominator in the pathogenesis of hyperglycemic injury [13-15] and diabetic kidney disease [14]. 8-hydroxy2 -deoxyguanosine (8-OHdG) is a product of DNA damage in the presence of high levels of ROS, with specific enzymatic cleavage and 8-hydroxylation of guanine bases. It is currently considered to be a biomarker of oxidative DNA damage in the pathogenesis of DN $[16,17]$. Generally, superoxide anion is rapidly removed by dismutation to hydrogen peroxide, which is decreased in diabetes $[18,19]$. This process occurs either spontaneously or is caused by superoxide dismutase (SOD). Few studies have reported an association between ROS and Ang-2 in type 2 diabetes patients with albuminuria.

In order to identify the factors associated with elevated Ang-2, we measured TNF- $\alpha$, IL18, 8-OHdG levels and SOD activity in the serum and urine of diabetic patients with normo-, micro-, or macroalbuminuria. We evaluated their relationship with Ang-2 to identify factors that might influence or predict Ang-2 levels. We aimed to determine whether there was an association between Ang-2, inflammatory cytokines (TNF- $\alpha$ and IL-18) and reactive oxygen species (8-OHdG and SOD) in type 2 diabetes patients with albuminuria.

\section{Materials and Methods}

\section{Subjects}

This retrospective study included 113 patients diagnosed with type-2 diabetes mellitus (T2DM). T2DM was defined by established WHO criteria [20], and the inclusion/exclusion criteria were same as in our previous study [5]. Briefly, the patients were recruited in the Department of Endocrinology and Nephrology at Union Hospital (Wuhan, China) between December 2012 and Mar 2014. Patients with macrovascular complications, acute diabetic complications, or other diseases that affect the urinary albumin excretion rate (UAER) were excluded. The patients were stratified into three groups by their UAER at enrollment: DN1 (UAER <20 $\mu \mathrm{g} / \mathrm{min})$; DN2 (20-200 $\mu \mathrm{g} / \mathrm{min})$; and DN3 ( $>200 \mu \mathrm{g} / \mathrm{min})$. Thirty healthy subjects were recruited as a control group (NC).

Ten patients had a diagnosis verified by pathological evaluation of a renal biopsy. Kidney specimens obtained from those patients were divided into two groups by 24-h proteinuria quantitative measurement (UPQM): DN-G1 (24-h UPQM, 0.5-3.5 g/d) and DN-G2 (24-h UPQM >3.5 g/d). Normal tissue obtained from 


\section{Kidney \\ Blood Pressure Research}

Kidney Blood Press Res 2015;40:355-365

\begin{tabular}{l|l}
\hline DOI: $10.1159 / 000368510$ & (C) 2015 S. Karger AG, Basel
\end{tabular}

Published online: June 20, 2015

www.karger.com/kbr

patients in the Urology Department of Union Hospital who underwent nephrectomy for renal carcinoma served as a control.

The Medical Ethics Committee of Union Hospital, Tongji Medical College, Huazhong University of Science and Technology approved the study protocol; the study was carried out following the ethical principles of the Declaration of Helsinki. Patients and control subjects gave their written, informed consent to participate in the study.

Enzyme-linked immunosorbent assay (ELISA) of Ang-2, TNF- $\alpha$, IL-18 and 8-OHdG

TNF- $\alpha$, IL-18, 8-OHdG, and Ang-2 levels in serum and urine were measured by an ELISA as we described previously [5]. Briefly, monoclonal antibodies specific for anti-Ang-2 (Raybiotech, Norcross, GA, USA), TNF- $\alpha$ (NeoBioscience Technology Co., Ltd, Shenzhen, China), IL-18 (Huaruikang Biotech Science Co., Ltd, Wuhan, China), and 8-OHdG (Cusabio Biotech Co., Ltd, Wuhan, China) were precoated onto microplates. Standards and samples obtained from study participants were added to the wells, and the Ang-2, TNF- $\alpha$, IL18 , and 8-OHdG present were bound by the immobilized antibody. After washing with phosphate buffered saline (PBS), biotinylated anti-human antibodies specific for Ang-2, TNF- $\alpha$, IL-18 or 8-OHdG were added. After washing unbound antibodies away, horseradish peroxidase (HRP)-conjugated streptavidin was added to the wells. After a final wash with PBS, a 3,3',5,5'-tetramethylbenzidine (TMB) substrate solution was pipetted into the wells and the intensity of the color that developed was measured with a microplate reader (BioTek ELX800, USA) at a wavelength of $450 \mathrm{~nm}$. All samples were tested in duplicate and the mean values were used for statistical analysis.

SOD activity detection

Total SOD activity in serum and urine were measured by a Superoxide Dismutase Assay Kit II (Calbiochem; Nanjing Jiancheng Bioengineering Institute, China) following the manufacturer's instructions. This assay uses a tetrazolium salt to detect superoxide radicals generated by xanthine oxidase and hypoxanthine. SOD activity was quantified by the hydroxylamine method using a spectrophotometer (SpectraMax 190 spectrophotometer, Molecular Devices Corporation, USA) at a wavelength of $550 \mathrm{~nm}$. One unit of SOD activity was defined as the amount of enzyme required for $50 \%$ dismutation of the superoxide radical [21], and SOD activity was expressed in $\mathrm{U} / \mathrm{ml}$.

\section{Immunohistochemistry}

Tissue samples were obtained by renal punch biopsy and prepared for immunohistochemical staining of TNF- $\alpha$, IL-18, 8-OHdG and NOX4. Normal tissue obtained following nephrectomy due to kidney carcinoma served as a control. Briefly, $3 \mu \mathrm{m}$ paraffin sections were deparaffinized in xylene and rehydrated in a $100 \%, 95 \%$, and $80 \%$ ethanol series followed by $0.1 \mathrm{~mol} / \mathrm{L} \mathrm{PBS}$, pH 7.4. Sections were then submerged in $10 \mathrm{mM}$ citrate buffer ( $\mathrm{pH} 6.0$ ), and antigen unmasking was completed in a microwave oven. After cooling, sections were immersed in $3 \% \mathrm{H}_{2} \mathrm{O}_{2}$ at room temperature for $10 \mathrm{~min}$, and in $5 \%$ bovine serum albumin (BSA) for $20 \mathrm{~min}$. Subsequently, sections were incubated overnight at $4^{\circ} \mathrm{C}$ with rabbit anti-IL-18, mouse anti-8-OHdG, goat anti-NOX4, or rabbit anti-TNF- $\alpha$ primary antibody (all from Santa Cruz Biotechnology, CA, USA). After washing, the specific secondary immunoglobulin was applied for 30 minutes at $37^{\circ} \mathrm{C}$. Colors were developed with diaminobenzidine (DAB, EnVision Detection Kit). Finally, sections were counterstained with hematoxylin and coverslipped using universal mounting medium. Control sections were obtained by omitting the primary antibody and replacing it with PBS.

\section{Statistical analysis}

Statistical analysis was done using SPSS, version 18.0, (SPSS, Chicago, IL). Data were presented as means \pm SEM. One-way analysis of variance (ANOVA) with Tukey's post hoc test was used to analyze normally distributed data. Kruskal-Wallis test was used to analyze non-normally distributed data. Pearson's or Spearman's correlation coefficients were used to test the significance of associations between variables. Factors that affected the two dependent outcome variables, serum Ang-2 and urinary Ang-2 levels, were determined by multivariable linear regression analysis including all those variables with $P \leq 0.15$ from univariate analysis by stepwise multiple regression. All tests were two-tailed. $P<0.05$ was considered statistically significant. 


\section{Kidney \\ Blood Pressure \\ Research}

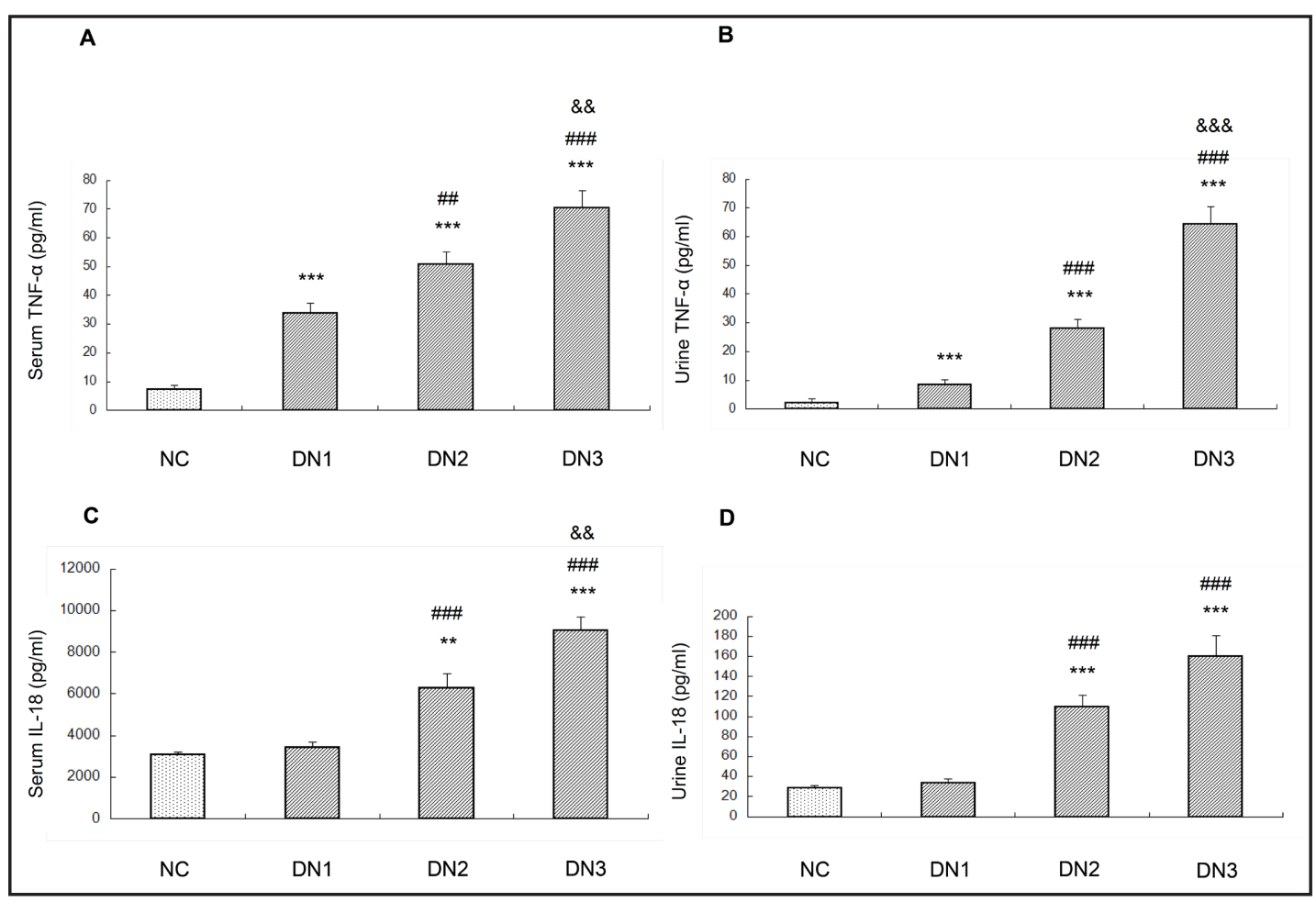

Fig. 1. Serum and urinary TNF- $\alpha$ and IL-18 levels in diabetic patients and controls. A. Serum and B. urine concentrations of TNF- $\alpha$ in diabetes patients and controls. The TNF- $\alpha$ concentrations showed stepwise increases in diabetes patients compared with controls according to the degree of albuminuria. C. Serum IL-18 levels were significantly increased in groups DN2 and DN3 compared with control subjects. D. Subjects with diabetes in groups DN2 and DN3 had significantly higher urinary IL-18 levels than control subjects. However, the increase from group DN2 to group DN3 did not reach significance. NC, control subjects without DN ; DN1, normoalbuminuria; DN2, microalbuminuria; DN3, macroalbuminuria. ${ }^{* *} P<0.01$ vs. NC; ${ }^{* * *} P<0.001$ vs.

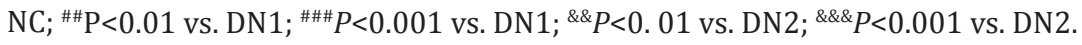

\section{Results}

TNF- $\alpha$ and IL-18 increased in serum and urine of type 2 diabetes patients

Clinically relevant patient characteristics and Ang-2 levels were described in our previous study report [5]. Serum and urine samples were collected from both the type 2 diabetes patients and healthy controls for determination of TNF- $\alpha$ and IL-18 concentrations. Serum TNF- $\alpha$ levels were significantly increased in diabetes patients compared with controls (Fig. 1A). When the concentrations in groups with different degrees of albuminuria were compared, it was found that serum TNF- $\alpha$ level was significantly higher in patients with macroalbuminuria (group DN3) than it was in groups DN1 and DN2 (Fig. 1A). Diabetes patients with normal albuminuria (group DN1) had higher levels of urinary TNF- $\alpha$ than controls (Fig. 1B), and urinary TNF- $\alpha$ increased in a stepwise manner with increasing degrees of albuminuria in the three groups of diabetes patients (Fig. 1B).

Unlike TNF- $\alpha$, the serum IL-18 level was not higher in group DN1 than in controls, but was higher in both patients with microalbuminuria and patients with macroalbuminuria (Fig. 1C). The increase in urinary IL-18 levels was similar to that observed in serum. The mean value was higher in group DN3 (macroalbuninuria) than in group DN2 (microalbuminuria), but the difference was not statistically significant (Fig. 1D). The expression of both TNF- $\alpha$ and IL-18 were increased in T2DM and peaked in group DN3; TNF- $\alpha$ was increased in patients with normoalbuminuria, but IL-18 began to increase in patients with microalbuminuria. 


\section{Kidney

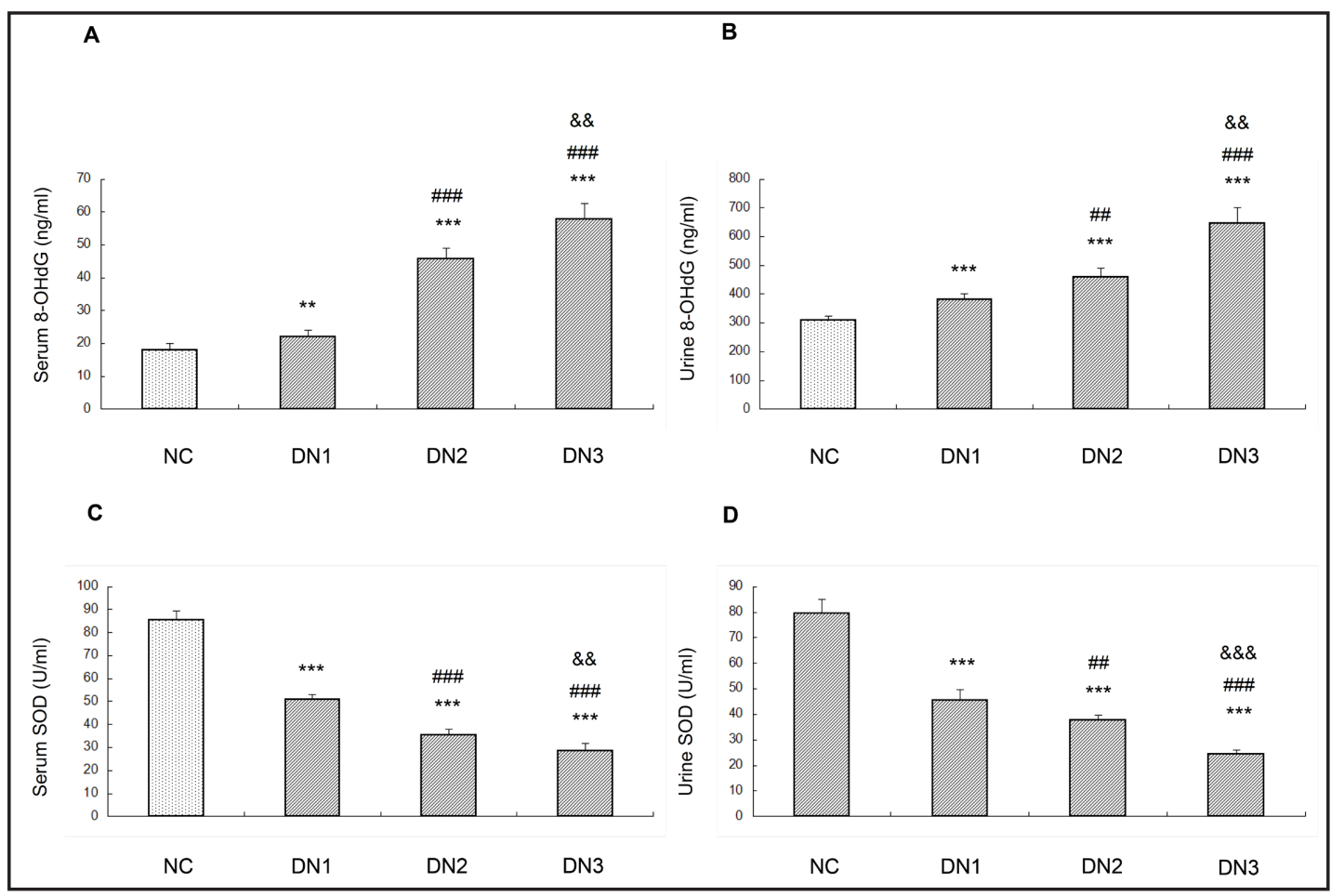

Fig. 2. Serum and urinary $8-\mathrm{OHdG}$ and SOD levels in diabetes patients and controls. A. Serum and B. urine concentrations of 8-OHdG in diabetic patients and controls. Urinary 8-OHdG levels showed stepwise increases in diabetic patients compared with controls according to the degree of albuminuria. C. Serum and D. urine SOD activity were significantly decreased beginning in group DN1 compared with control subjects. NC, control subjects without DN ; DN1, normoalbuminuria; DN2, microalbuminuria; DN3, macroalbuminuria. ** $\mathrm{P}<0.01$ vs. NC; ${ }^{* * *} P<0.001$ vs. NC; ${ }^{\#} P<0.01$ vs. DN1; ${ }^{\# \# ~} P<0.001$ vs. DN1; ${ }^{\&} P<0.01$ vs. DN2; \&\&\& $P<0.001$ vs. DN2.

8-OHdG increased and SOD activity decreased in serum and urine of type 2 diabetes patients

8-OHdG concentration and SOD activity were assayed in the serum and urine of T2DM patients to determine oxidant and antioxidant capacity. The serum concentration of 8-OHdG was significantly increased in diabetes patients compared with controls and increased significantly with each degree of albuminuria (Fig. 2A). The concentration of 8-0HdG in the urine of diabetes patients was also significantly increased compared with controls and increased significantly with each degree of albuminuria (Fig. 2B).

In contrast to 8-OHdG, SOD activity was significantly decreased in the serum and urine of diabetes patients compared with the control group. Moreover, serum and urine SOD activity both decreased significantly with each stepwise increase of albuminuria (Fig. 2C, D). Thus we found that 8-OHdG, which represents oxidant capacity, increased with the degree of albuminuria in diabetes patients who had baseline normoalbuminuria. At the same time, and in the same patients, SOD activity, a marker of the antioxidant capacity, decreased.

Increased TNF- $\alpha, I L-18,8-O H d G$, and NOX4 expression in glomeruli of diabetes patients

The expressions of TNF- $\alpha$, IL-18, 8-OHdG and NOX4 in kidney glomeruli of diabetes patients was evaluated by immunohistochemical staining of renal punch biopsy tissues. As shown in Fig. 3, strong staining of TNF- $\alpha$, IL-18, 8-OHdG and NOX4 protein was detected in glomeruli alongside capillary loops of group DN-G1 patients, which to some extent reflects early changes of DN. In group DN-G2 patients, most glomeruli had lost the characteristic 


\section{Kidney Blood Pressure Research}

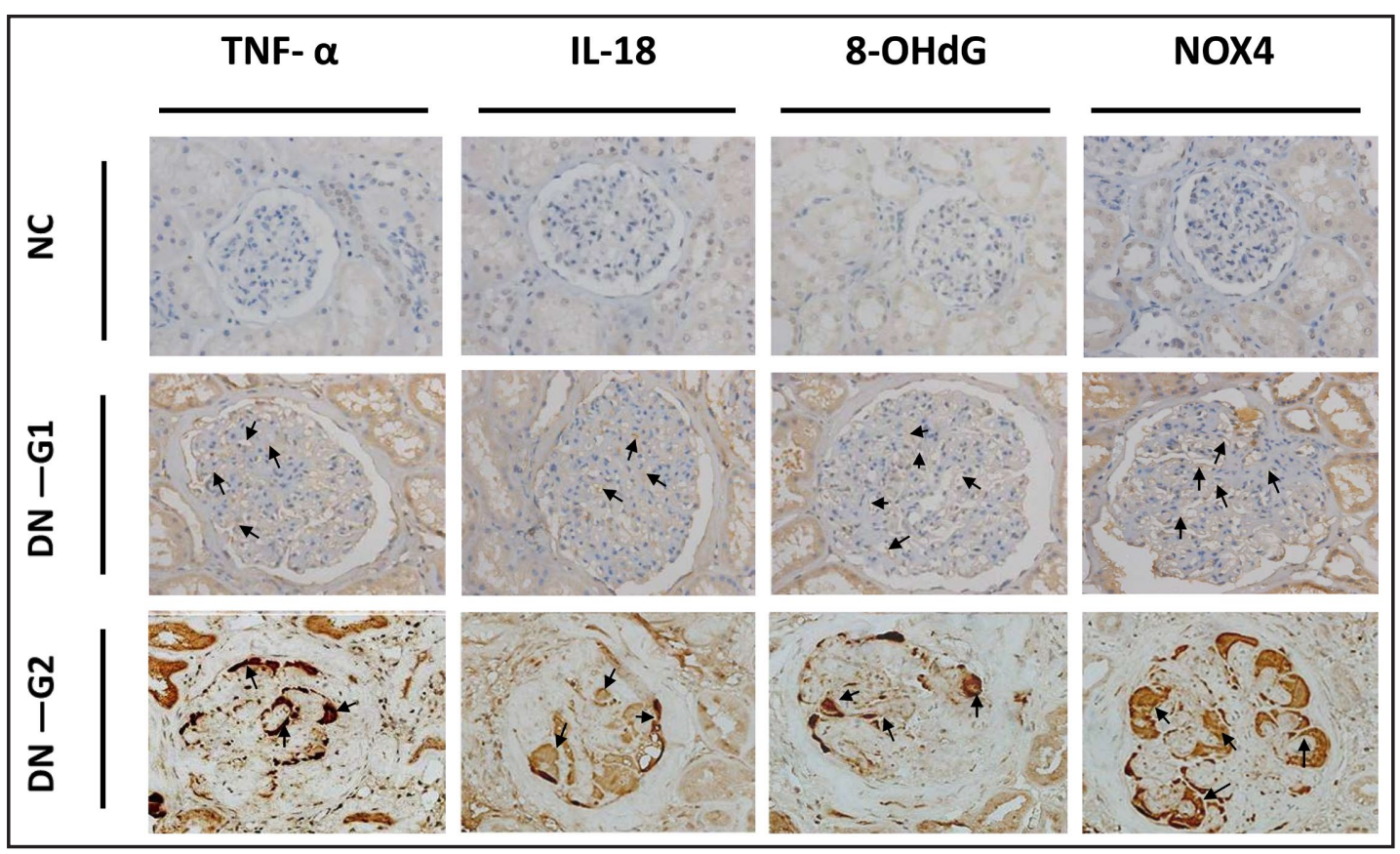

Fig. 3. Increased expression of TNF- $\alpha$, IL-18, 8-OHDG and NOX4 in the glomeruli of diabetic patients. TNF- $\alpha$, IL-18, 8-OHDG and NOX4 staining was not detectable in the glomeruli of control subjects without DN (NC, $400 \times$ magnification). Representative images of renal biopsy sections from patients from group DN-G1 showed abundant staining of TNF- $\alpha$, IL-18, 8-OHdG and NOX4 protein in glomeruli alongside capillary loops. In group DN-G2, most glomeruli had lost the fundamental vascular and basement-membrane structure with progression to tuberous sclerosis lesions, staining was enhanced, with sparse accumulation of positive cells in the in extracellular matrix and mesangium. NC, control subjects without DN; DN-G1, DN with $24 \mathrm{~h}$ UPQM from 0.5-3.5 g/d; DN-G2, diabetic nephropathy with 24h UPQM >3.5 g/d. Arrows indicate positively stained cells.

vascular and basement membrane structure with progression to tuberous sclerosis lesions; staining was enhanced, with sparse accumulation of positive cells in the extracellular matrix and mesangium. No positively stained cells were observed in glomeruli of normal control tissue.

\section{Correlation and multivariable linear regression analysis}

Figs. 4 and 5 show the scatter plots and correlation coefficients for Ang- 2 with the markers described above and calculated for in all diabetes patients. The Ang-2 levels in serum and urine were described in our previous study [5]. Correlation analysis showed the serum level of Ang-2 was positively correlated with serum levels of TNF- $\alpha(P<0.001)$, IL$18(P<0.001), 8-0 H d G(P<0.001)$, urine levels of TNF- $\alpha(P<0.001)$, IL-18 $(P<0.001), 8-0 H d G$ $(P<0.001)$; and negatively correlated with SOD activity of serum and urine (both $P<0.001$, Fig. 4). Urinary Ang-2 concentration was significantly positively correlated with serum levels of TNF- $\alpha(P<0.001)$, IL-18 $(P<0.001), 8-0 H d G(P<0.001)$, urine levels of TNF- $\alpha(P<0.001)$, IL$18(P<0.001), 8-0 H d G(P<0.001)$; and negatively correlated with both serum and urine SOD activity (both $P<0.001$, Fig. 5). Multivariable linear regression analysis revealed that serum TNF- $\alpha$ (standardized regression coefficient $\beta=0.448, P<0.001$ ), $8-0 H d G(\beta=0.180, P=0.001$ ), and IL-18 ( $\beta=0.173, P=0.003)$ were independent predictors of serum Ang-2 level (with serum TNF- $\alpha$, IL-18, 8-OHdG levels and SOD activity as independent variables). Urinary TNF- $\alpha$ ( $=0.674, P<0.001)$ and $8-0 H d G(\beta=0.135, P=0.004)$ were independent predictors of urinary Ang-2 level (with urinary TNF- $\alpha$, IL-18, 8-OHdG and SOD as independent variables). 


\section{Kidney \\ Blood Pressure \\ Research}

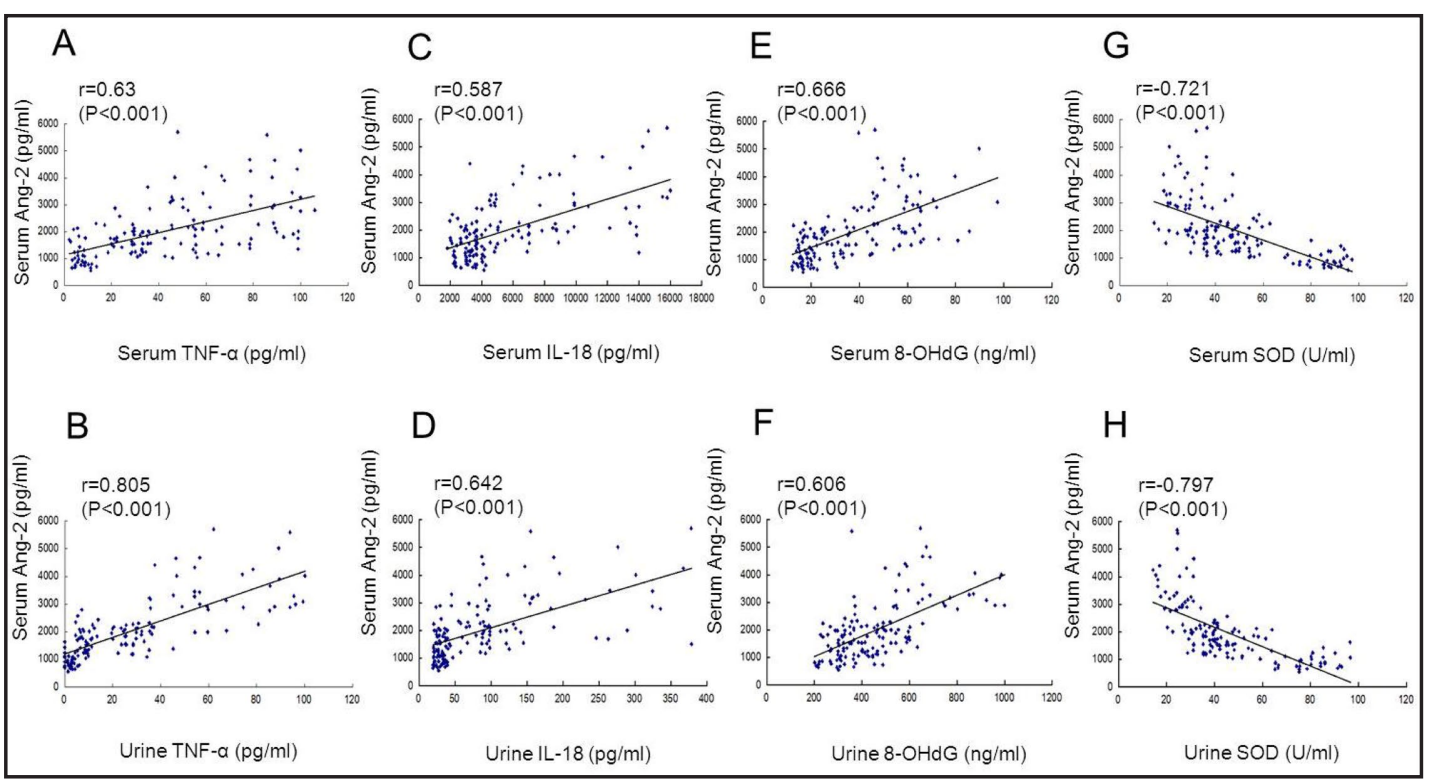

Fig. 4. Correlation analysis for serum Ang-2 and TNF- $\alpha$, IL-18, 8-OHDG, and SOD. Serum Ang-2 level correlated positively with serum and urinary TNF- $\alpha$ (A, B), IL-18 (C, D), 8-OHdG (E, F) and negatively with SOD $(G, H)$. TNF- $\alpha$, tumor necrosis factor- $\alpha$; IL-18, interleukin 18; 8-OHdG, 8-hydroxy-2'-deoxy guanosine; SOD, superoxide dismutase.

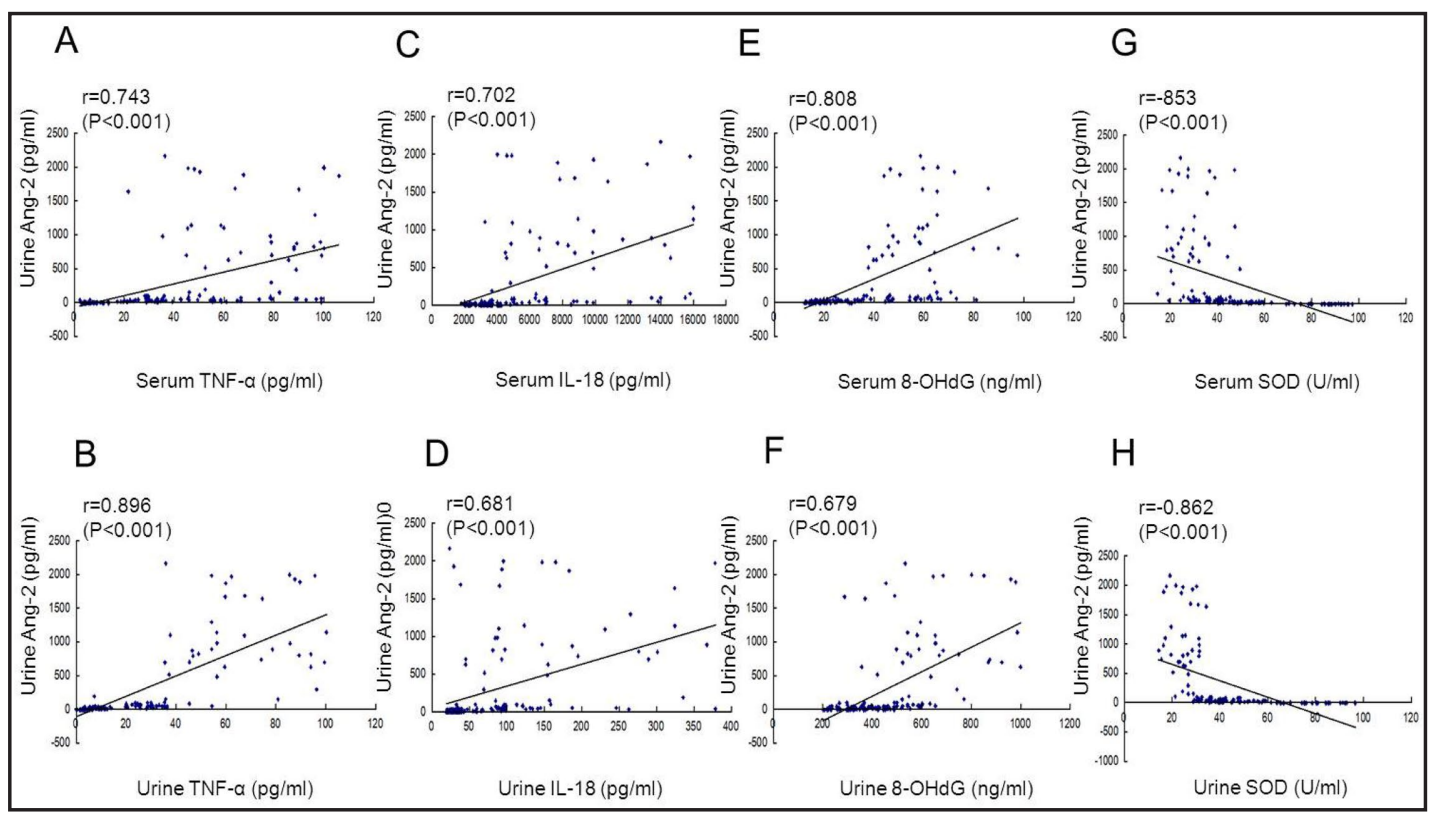

Fig. 5. Correlation analysis for urinary Ang-2 and TNF- $\alpha$, IL-18, 8-OHDG, and SOD. Urinary Ang-2 level correlated positively with serum and Urinary TNF- $\alpha$ (A, B), IL-18 (C, D) 8-OHdG (E, F) and negatively with $\operatorname{SOD}(G, H)$. TNF- $\alpha$, tumor necrosis factor- $\alpha$; IL-18, Interleukin 18; 8-OHdG, 8-hydroxy-2'-deoxy guanosine; SOD, Superoxide dismutase.

\section{Discussion}

In this study, we found that TNF- $\alpha$, IL-18 and 8-OHdG concentrations in the serum and urine of diabetes patients increased with albuminuria, and were positively correlated with 


\section{Kidney Blood Pressure Research}

Kidney Blood Press Res 2015;40:355-365

\begin{tabular}{l|l}
\hline DOI: $10.1159 / 000368510$ & (C) 2015 S. Karger AG, Basel
\end{tabular}

Published online: June 20, 2015

www.karger.com/kbr

increased Ang-2 levels. The TNF- $\alpha$ and 8-OHdG concentrations were higher in patients with normoalbuminuria than in control patients. Conversely, SOD activity in both serum and urine decreased with the development of albuminuria, and was negatively correlated with Ang-2. Furthermore, serum levels of TNF- $\alpha, 8-\mathrm{OHdG}$, and IL-18 predicted serum levels of Ang-2. Urinary Ang-2 level was independently associated with urinary TNF- $\alpha$ and 8-OHdG.

TNF- $\alpha$ and IL-18 are both prominent pro-inflammatory cytokines, and our study suggests that TNF- $\alpha$ may be implicated in the pathogenesis of DN because it was already increased in diabetes patients without overt albuminuria. TNF- $\alpha$ and IL-18 may reflect early renal dysfunction in patients with T2DM because they were observed in the presence of microalbuminuria. Positive immunohistochemical staining of TNF- $\alpha$ and IL-18 in the glomeruli of diabetes patients is consistent with previous studies that found overexpression of TNF- $\alpha$ mRNA or protein and IL-18 protein in glomeruli and renal tubules of humans and in animal models of diabetes [22-24]. Overall, these studies demonstrate that increases in TNF- $\alpha$ and IL-18 are associated with renal damage and may facilitate the progress of DN.

The significant positive correlation of increase in Ang- 2 with increase in both TNF- $\alpha$ and IL-18 indicates that the renal dysfunction caused by hyperglycemia in DN might, at least partly, be due to interactions of TNF- $\alpha$ and IL-18 with Ang-2. Our study results suggest that Ang- 2 and TNF- $\alpha$ might both be considered as early biomarkers of DN as they began to increase before microalbuminuria could be detected.

TNF- $\alpha$ level is an independent predictor of both serum Ang-2 and urinary Ang-2. Fiedler et al. reported the rapid release of Ang-2 from Weibel-Palade bodies, with induction of inflammation following TNF- $\alpha$ release in response to exogenous stimuli [25]. It has also been shown that the expression of Ang-2 mRNA is increased in the presence of TNF- $\alpha$ (10 ng/ $\mathrm{ml}$ ) [26]. The results of this study together with the above lead to the suggestion that, in the presence of hyperglycemia, TNF- $\alpha$ might induce not only an inflammatory response, but also an angiogenic response involving overexpression of Ang-2. However, Yao et al. demonstrated that Ang-2 induced by hyperglycemia was sufficient to sensitize microvascular endothelial cells to TNF- $\alpha$ by its proinflammatory effects [27]. It is thus possible that Ang- 2 release might enhance the harmful effects of TNF- $\alpha$ to induce an inflammatory cascade facilitating the development of DN. However, additional studies are needed to determine the specific causeeffect relationships among these biomarkers of DN.

It is well established that ROS is a common denominator in the pathogenesis of DN. Increased ROS activity can result from increased oxidant production and/or decreased antioxidant function. 8-OHdG, is generally regarded as a marker of oxidative DNA damage [28], and is produced in the presence of high levels of ROS. SOD, known as the superoxide scavenger enzyme, constitutes the first line of defense against ROS. In this study, 8-OHdG levels were elevated and SOD activity was decreased in the serum and urine of type 2 diabetes patients. This is consistent with previous reports that 8-OHdG levels are increased in diabetes patients and in animal models and that reduced SOD activity is involved in the pathology of diabetes and DN [28-31].

In addition, changes in 8-OHdG level and SOD activity occurred in parallel with the severity of albuminuria. Immunohistochemical staining of NOX4, which is thought to be a major contributor to renal ROS [32], and 8-OHdG were both positive in the endothelium of the glomerulus and vascular lumen in the early stage of DN and strongly positive in glomeruli that had lost the fundamental vascular and basement-membrane structure. These findings indicated that oxidative damage due to an imbalance of oxidant and antioxidant activity already existed in the early stage of DN, and was associated with renal injury. The 8 -OHdG level was nearly 10 -fold higher in urine than in serum, which together with the immunohistochemistry results leads us to infer the urinary 8-OHdG might derive from increased systemic oxidative stress due to NOX4-mediated ROS in renal tissue.

Furthermore, 8-OHdG levels were positively correlated with increased Ang-2 in both the serum and urine of type 2 diabetes patients; $8-0 H d G$ was an independent predictor 


\section{Kidney \\ Blood Pressure Research}

Kidney Blood Press Res 2015;40:355-365

\begin{tabular}{l|l}
\hline DOI: $10.1159 / 000368510$ & (c) 2015 S. Karger AG, Basel
\end{tabular}

Published online: June 20, 2015

www.karger.com/kbr

of Ang-2 levels in serum and urine. This relationship between Ang-2 and 8-OHdG has not been reported previously. Growing evidence reveals that ROS produced by NOX act as signal molecules in angiogenesis to promote diverse redox signaling pathways and lead to the induction of angiogenesis-related genes, endothelial growth and proliferation [33]. In a study of oxidative damage of lung tissue, Faller et al. reported that hydrogen sulfide could simultaneously downregulate ROS and Ang-2 [34]. We thus propose that Ang-2 might be involved in the process of ROS-promoted angiogenesis. It has been reported that Ang-2 secretion and its effect on promoting vascular survival is regulated by extracellular regulating kinase (ERK), Jun N-terminal kinase (JNK), phosphatidylinositol 3-kinase (PI3kinase), and eNOS pathways. These pathways have also been reported to play important roles upstream and downstream of ROS [35-37], which potentially supports our conclusion that 8-OHdG is involved in the elevation of serum and urine Ang-2 concentrations in DN. Our results warrant additional study of how 8-OHdG and Ang-2 influence the progression of DN.

\section{Study limitations}

The findings of this study should be interpreted in the light of some limitations. First, this was a single-center, cross-sectional study that enrolled a small population. Second, we could only demonstrate an association between Ang- 2 and inflammatory factors (TNF- $\alpha$, IL18), or Ang-2 and ROS (8-OHdG, SOD), not a causal relationship. The molecular mechanisms responsible for increased Ang- 2 in DN can at first be investigated In vitro and in vivo in animal models. Third, in the statistical analysis, we did not adjust for possible confounders that may have affected the associations of TNF- $\alpha$, IL-18, 8-OHdG, or SOD with Ang-2 levels. Because the study included only diabetes patients, we could not evaluate the precise effects of the confounders in this complex pathophysiological environment.

\section{Conclusion}

Increased levels of TNF- $\alpha$, IL-18, and 8-OHdG and decreased SOD activity were associated with a worsening of albuminuria. Serum TNF- $\alpha$, IL-18, and 8-OHdG predicted serum Ang-2 levels; urinary TNF- $\alpha$ and 8-OHdG predicted urinary Ang-2 levels. TNF- $\alpha$ and 8-OHdG were associated with elevated urinary Ang-2 levels in type 2 diabetes patients with albuminuria.

\section{Disclosure Statement}

The authors have no conflicts of interest to declare.

\section{Acknowledgements}

This work was supported by grants from the National Natural Science Foundation of China (No. 30871174, No. 30800523, No. 30900682, No. 81170662, No. 81170600, No. 31200872, No. 81300604, No. 81470964 and No. 81400720), the Natural Science Foundation of Hubei Province (No. 2013 CFA026 and No. 2012 FFA038), the Doctoral Fund of the Ministry of Education of China (No. 20130142110064), a grant from Hubei Province Health and Family Planning Commission (No. WJ2015MB013), and a grant from the Science and Technology Bureau of Wuhan (No. 2015060101010039). 


\section{Kidney \\ Blood Pressure Research}

\section{References}

1 Woolf AS: Angiopoietins: Vascular growth factors looking for roles in glomeruli. Curr Opin Nephrol Hypertens 2010;19:20-25.

2 Martynov SA, Shestakova MV, Kutyrina IM, Il'in AV, Arbuzova MI, Dedov, II: [role of circulating angiogenic factors in diabetic kidney disease]. Vestn Ross Akad Med Nauk 2013:35-42.

3 de Caestecker MP: Angiopoietin-2 and glomerular proteinuria. J Am Soc Nephrol 2007;18:2217-2218.

4 Chang FC, Lai TS, Chiang CK, Chen YM, Wu MS, Chu TS, Wu KD, Lin SL: Angiopoietin-2 is associated with albuminuria and microinflammation in chronic kidney disease. PLoS One 2013;8:e54668.

5 Chen S, Li H, Zhang C, Li Z,Wang Q Guo J, Luo C, Wang Y: Urinary Angiopoietin-2 is Associated with Albuminuria in Patients with Type 2 Diabetes Mellitus. Int J Endocrinol 2015;2015:163120.

6 Fiedler U, Augustin HG: Angiopoietins: A link between angiogenesis and inflammation. Trends Immunol 2006;27:552-558.

7 Jackson JR, Seed MP, Kircher CH, Willoughby DA, Winkler JD: The codependence of angiogenesis and chronic inflammation. FASEB J 1997;11:457-465.

8 Dvorak HF, Nagy JA, Feng D, Brown LF, Dvorak AM: Vascular permeability factor/vascular endothelial growth factor and the significance of microvascular hyperpermeability in angiogenesis. Curr Top Microbiol Immunol 1999;237:97-132.

9 Navarro-Gonzalez JF, Mora-Fernandez C, Muros de Fuentes M, Garcia-Perez J: Inflammatory molecules and pathways in the pathogenesis of diabetic nephropathy. Nat Rev Nephrol 2011;7:327-340.

10 Moriwaki Y, Yamamoto T, Shibutani Y, Aoki E, Tsutsumi Z, Takahashi S, Okamura H, Koga M, Fukuchi M, Hada T: Elevated levels of interleukin-18 and tumor necrosis factor-alpha in serum of patients with type 2 diabetes mellitus: Relationship with diabetic nephropathy. Metabolism 2003;52:605-608.

11 Navarro-Gonzalez JF, Jarque A, Muros M, Mora C, Garcia J: Tumor necrosis factor-alpha as a therapeutic target for diabetic nephropathy. Cytokine Growth Factor Rev 2009;20:165-173.

12 Luo C, Li T, Zhang C, Chen Q Li Z, Liu J, Wang Y: Therapeutic effect of alprostadil in diabetic nephropathy: Possible roles of angiopoietin-2 and il-18. Cell Physiol Biochem 2014;34:916-928.

13 Kanwar YS, Wada J, Sun L, Xie P, Wallner EI, Chen S, Chugh S, Danesh FR: Diabetic nephropathy: Mechanisms of renal disease progression. Exp Biol Med (Maywood) 2008;233:4-11.

14 Brownlee M: The pathobiology of diabetic complications: A unifying mechanism. Diabetes 2005;54:16151625.

15 Kumpers P, Koenecke C, Hecker H, Hellpap J, Horn R, Verhagen W, Buchholz S, Hertenstein B, Krauter J, Eder M, David S, Gohring G, Haller H, Ganser A: Angiopoietin-2 predicts disease-free survival after allogeneic stem cell transplantation in patients with high-risk myeloid malignancies. Blood 2008;112:2139-2148.

16 Kanauchi M, Nishioka H, Hashimoto T: Oxidative DNA damage and tubulointerstitial injury in diabetic nephropathy. Nephron 2002;91:327-329.

17 Xu GW, Yao QH, Weng QF, Su BL, Zhang X, Xiong JH: Study of urinary 8-hydroxydeoxyguanosine as a biomarker of oxidative DNA damage in diabetic nephropathy patients. J Pharm Biomed Anal 2004;36:101104.

18 Chattopadhyay M, Khemka VK, Chatterjee G, Ganguly A, Mukhopadhyay S, Chakrabarti S: Enhanced ros production and oxidative damage in subcutaneous white adipose tissue mitochondria in obese and type 2 diabetes subjects. Mol Cell Biochem 2015;399:95-103.

19 Matyal R, Sakamuri S, Huang T, Owais K, Parikh S, Khabbaz K, Wang A, Sellke F, Mahmood F: Oxidative stress and nerve function after cardiopulmonary bypass in patients with diabetes. Ann Thorac Surg 2014;98:1635-1643.

20 Alberti KG, Zimmet PZ: Definition, diagnosis and classification of diabetes mellitus and its complications. Part 1: Diagnosis and classification of diabetes mellitus provisional report of a who consultation. Diabet Med 1998;15:539-553.

21 Ozbal S, Ergur BU, Erbil G, Tekmen I, Bagriyanik A, Cavdar Z: The effects of alpha-lipoic acid against testicular ischemia-reperfusion injury in rats. ScientificWorldJournal 2012;2012:489248. 


\section{Kidney \\ Blood Pressure Research}

\section{Kidney Blood Press Res 2015;40:355-365}

\begin{tabular}{l|l}
\hline DOI: $10.1159 / 000368510$ & (C) 2015 S. Karger AG, Basel
\end{tabular}

Published online: June 20, 2015

www.karger.com/kbr

22 Sugimoto H, Shikata K, Wada J, Horiuchi S, Makino H: Advanced glycation end products-cytokine-nitric oxide sequence pathway in the development of diabetic nephropathy: Aminoguanidine ameliorates the overexpression of tumour necrosis factor-alpha and inducible nitric oxide synthase in diabetic rat glomeruli. Diabetologia 1999;42:878-886.

23 Navarro JF, Milena FJ, Mora C, Leon C, Claverie F, Flores C, Garcia J: Tumor necrosis factor-alpha gene expression in diabetic nephropathy: Relationship with urinary albumin excretion and effect of angiotensinconverting enzyme inhibition. Kidney Int Suppl 2005:S98-102.

24 Miyauchi K, Takiyama Y, Honjyo J, Tateno M, Haneda M: Upregulated il-18 expression in type 2 diabetic subjects with nephropathy: Tgf-beta1 enhanced il-18 expression in human renal proximal tubular epithelial cells. Diabetes Res Clin Pract 2009;83:190-199.

25 Fiedler U, Reiss Y, Scharpfenecker M, Grunow V, Koidl S, Thurston G, Gale NW, Witzenrath M, Rosseau S, Suttorp N, Sobke A, Herrmann M, Preissner KT, Vajkoczy P, Augustin HG: Angiopoietin-2 sensitizes endothelial cells to tnf-alpha and has a crucial role in the induction of inflammation. Nat Med 2006;12:235239.

26 Kim I, Kim JH, Ryu YS, Liu M, Koh GY: Tumor necrosis factor-alpha upregulates angiopoietin-2 in human umbilical vein endothelial cells. Biochem Biophys Res Commun 2000;269:361-365.

27 Yao D, Taguchi T, Matsumura T, Pestell R, Edelstein D, Giardino I, Suske G, Rabbani N, Thornalley PJ, Sarthy VP, Hammes HP, Brownlee M: High glucose increases angiopoietin-2 transcription in microvascular endothelial cells through methylglyoxal modification of msin3a. J Biol Chem 2007;282:31038-31045.

28 Shao N, Kuang HY, Wang N, Gao XY, Hao M, Zou W, Yin HQ: Relationship between oxidant/antioxidant markers and severity of microalbuminuria in the early stage of nephropathy in type 2 diabetic patients. J Diabetes Res 2013;2013:232404.

29 Li YS, Song MF, Kasai H, Kawai K: 8-hydroxyguanine in urine and serum as an oxidative stress marker: Effects of diabetes and aging. J UOEH 2013;35:119-127.

30 Shin CS, Moon BS, Park KS, Kim SY, Park SJ, Chung MH, Lee HK: Serum 8-hydroxy-guanine levels are increased in diabetic patients. Diabetes Care 2001;24:733-737.

31 Lim JH, Youn DY, Yoo HJ, Yoon HH, Kim MY, Chung S, Kim YS, Chang YS, Park CW, Lee JH: Aggravation of diabetic nephropathy in bcl-2 interacting cell death suppressor (bis)-haploinsufficient mice together with impaired induction of superoxide dismutase (sod) activity. Diabetologia 2014;57:214-223.

32 Sedeek M, Montezano AC, Hebert RL, Gray SP, Di Marco E, Jha JC, Cooper ME, Jandeleit-Dahm K, Schiffrin EL, Wilkinson-Berka JL, Touyz RM: Oxidative stress, nox isoforms and complications of diabetes--potential targets for novel therapies. J Cardiovasc Transl Res 2012;5:509-518.

33 Kim YW, Byzova TV: Oxidative stress in angiogenesis and vascular disease. Blood 2014;123:625-631.

34 Faller S, Spassov SG, Zimmermann KK, Ryter SW, Buerkle H, Loop T, Schmidt R, Strosing KM, Hoetzel A: Hydrogen sulfide prevents hyperoxia-induced lung injury by downregulating reactive oxygen species formation and angiopoietin-2 release. Curr Pharm Des 2013;19:2715-2721.

35 Wu WS, Wu JR, Hu CT: Signal cross talks for sustained mapk activation and cell migration: The potential role of reactive oxygen species. Cancer Metastasis Rev 2008;27:303-314.

36 Lee CC, Chen SC, Tsai SC, Wang BW, Liu YC, Lee HM, Shyu KG: Hyperbaric oxygen induces vegf expression through erk, jnk and c-jun/ap-1 activation in human umbilical vein endothelial cells. J Biomed Sci 2006;13:143-156.

37 Harfouche R, Hussain SN: Signaling and regulation of endothelial cell survival by angiopoietin-2. Am J Physiol Heart Circ Physiol 2006;291:H1635-1645. 